\title{
Dopamine Deficiency-Its Potential Contribution to Chronic Renal Failure Complicating Hypertension
}

\author{
Otto Kuchel, and Shuichi Shigetomi
}

\begin{abstract}
Baseline dihydroxyphenylalanine (DOPA) and dopamine (DA), their respective sulfates as well as oral DOPA administration-induced changes were compared in age- and blood pressure-matched hypertensive patients without and with moderate chronic renal failure (CRF) and control subjects. The only common feature of both hypertensive groups was a defective DA generation from DOPA. Hypertensive patients with moderate CRF were distinct from those without, having increased basal concentrations of plasma DOPA and DA sulfates. After oral DOPA administration, plasma and urinary DOPA sulfate rose while renal DA sulfate clearance was decreased. Possible enzymatic defects contributing to CRF-induced increases of DOPA and DA sulfates and their potential role in perpetuating renal failure via glomerular hypertension are discussed. (Hypertens Res 1995; 18 Suppl. I: S183-S185)
\end{abstract}

Key Words: dopamine, renal failure, glomerular hypertension

The vasodilating and natriuretic actions of dopamine (DA) appear to be an important part of the antihypertensive "defense" system in hypertension (HBP), preventing also one of its main complications, progressive renal failure. Glomerular hypertension is considered to be a cause and consequence of renal injury (1). DA may be a factor preventing it by its predominant vasodilating action on the vas efferents (2). The dichotomy of dopaminergic indices in hypertensive patients without and with chronic renal failure (CRF) may clarify the role of DA deficiency in HBP complicated by renal failure. DA is generated by dihydroxyphenylalanine (DOPA) decarboxylation in several neuronal and nonneuronal tissues, particularly the kidney. In previous studies, we therefore used an oral precursor DOPA load in an attempt to at least partially reproduce patterns of DA generation by DOPA decarboxylation $(3,4)$. We paid particular attention to DOPA and DA sulfoconjugation since an increase in sulfoconjugates dominates CRF (5), affecting the availability of free DA and its biological action.

\section{Methods and Results}

Twenty-two comparably hypertensive patients (12 without and 10 with moderate CRF defined by a glomerular filtration rate between 20 and 40 $\mathrm{mL} / \mathrm{min}$ ) and 5 normotensive controls, all Caucasians, age-matched, off medication and on a controlled diet, with a comparable state of sodium balance received $500 \mathrm{mg}$ of oral DOPA. Baseline sampling was followed by 3 hourly measurements of plasma and urinary DOPA and its sulfate by HPLC and of free DA and DA sulfate radioenzymatically.
The data were evaluated by multiple analysis of variance with repeated measures in 1 fraction for DOPA-induced modifications; non-parametric tests were used where justified. All details of the protocol, methods and analysis of data were previously described $(3,4)$.

One of the simplest indices of DOPA decarboxylation to DA is the plasma DOPA:DA ratio prior to and at 3 hourly periods after DOPA administration (Fig. 1). At baseline, there was no difference between the 3 groups. At $60 \mathrm{~min}$ and $120 \mathrm{~min}$ after L-DOPA administration, the ratio was comparable in the hypertensive groups with and without CRF, suggesting slower DOPA decarboxylation to DA in both since their ratios were higher than in the controls.

The most striking baseline difference between the 3 groups was the increased $\left(p<0.05^{*}\right)$ concentration of plasma DOPA sulfate in $\mathrm{HBP}$ with $\mathrm{CRF}$ $(7.8 \pm 3.2 \mathrm{pmol} / \mathrm{ml}$ * compared to $0.3 \pm 0.01$ in HBP and $1.29 \pm 0.9$ in the controls) and of plasma DA sulfate in HBP with CRF $\left(20.1 \pm 3.2 \mathrm{pmol} / \mathrm{ml}^{*}\right.$ compared to $6.8 \pm 1.2$ in HBP and $9.2 \pm 2.1$ in the controls). Most evident was the extremely high DOPA sulfate level in patients with HBP + CRF 60 min and $120 \mathrm{~min}$ following DOPA administration $(156 \pm 58 \mathrm{pmol} / \mathrm{ml}$ at $60 \mathrm{~min}$ and $160 \pm 36$ at 120 min) while it remained almost undetectable in HBP and the controls. DOPA sulfate appeared in plasma in HBP only after a delay (180 min after DOPA administration) with values comparable to those of $\mathrm{HBP}+\mathrm{CRF}$ but was always undetectable in the controls. In contrast, the overall increase of DA sulfate in all 3 groups following DOPA administration remained comparable. 


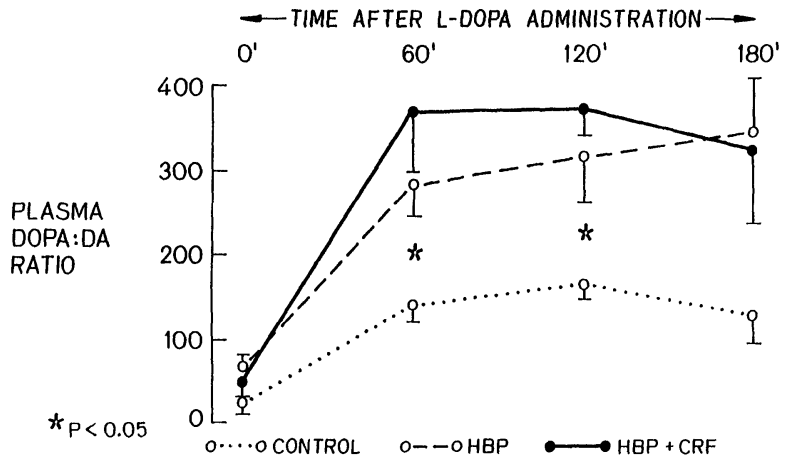

Fig. 1. Plasma free DOPA: free DA ratio prior to and $60 \mathrm{~min}, 120 \mathrm{~min}$ and $180 \mathrm{~min}$ following $L-D O P A$ administration in two HBP (with or without $C R F$ ) groups and in control patients.
The urinary excretory patterns again highlighted the elevation of urinary DOPA sulfate $1 \mathrm{~h}$ and $3 \mathrm{~h}$ after DOPA administration in HBP + CRF (1653 \pm $910 \mu \mathrm{mol} / \mathrm{h}^{*}$ and $2260 \pm 905 *$ respectively) but DA sulfate excretion in the 2 -h post-DOPA period was significantly lower in $\mathrm{HBP}+\mathrm{CRF}$ than in HBP and the controls $\left(17.6 \pm 2.8^{*} \mu \mathrm{mol} / \mathrm{h}\right.$ vs. $44.5 \pm 9.2$ and $61.7 \pm 14.4$ respectively).

\section{Discussion}

Those data suggest that HBP patients with CRF exhibit baseline differences, some of them accentuated by dynamic DOPA challenge. The baseline elevation of plasma DOPA sulfate in CRF is a new finding while that of DA sulfate has already been reported $(5,6)$. After DOPA administration, the increase of DOPA sulfate again predominates in HBP $+\mathrm{CRF}$ and is followed by augmented renal DOPA excretion, while it appears with a delay in hyperten-

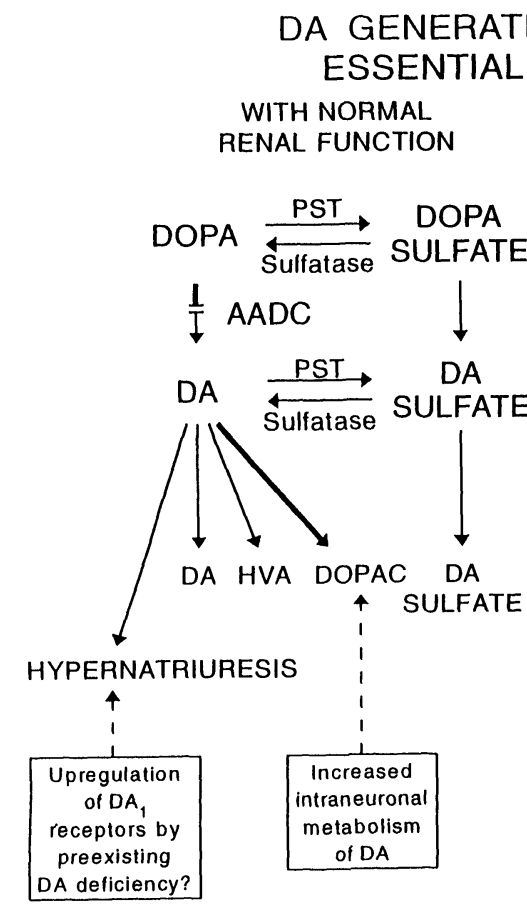

Hypertension 19:634-8, 1992

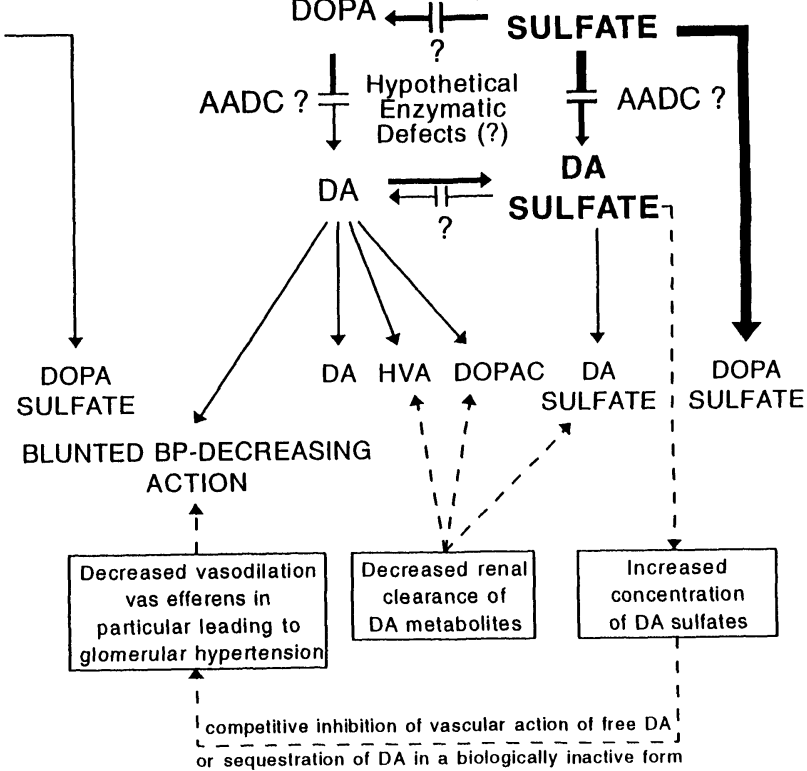

Hypertension 23:1 240-5, 1994

Fig. 2. Summary of data and previous results $(3,4)$, their interpretation and impact on the role of $D A$ in $H B P$ without and with CRF. In the presence of normal renal function (left), DA generation from DOPA is defective with a hypernatriuretic response to DOPA and indications of increased intraneuronal DA metabolism. With moderate CRF complicating HBP (right), sulfoconjugated DOPA and DA concentrations are elevated. This is associated with a blunted BP-decreasing action of DOPA administration (despite comparable free DA generation) and diminished renal clearance of DA metabolites (HVA, DOPAC and DA sulfates). Increased DA sulfoconjugates may inhibit the vasodilating effect of $D A$ (or sequester free $D A$ in a biologically-inactive form) and perpetuate $C R F$ by promoting glomerular hypertension. The DOPA sulfate excess over DA sulfate may be due to AADC suppression; circumstantial evidence indicates that an excess of DOPA and DA sulfates over their free precursors may be due to sulfatase inhibition (13). Both enzymes may be inhibited by a decrease of their main source, i.e. renal cellular mass (10) (or by uremic toxins?). AADC: Aromatic acid decarboxylase; PST: Phenolsulfotransferase; HVA: Homovanillic acid; DOPAC: Dopacetic acid. 
sives without CRF but not in the controls. However, there is no elevation of plasma DA sulfate in hypertensives with CRF compared with the other groups and DA sulfate clearance is decreased. This indicates different renal excretory mechanisms for DOPA sulfate and DA sulfate, the fractional excretion of DOPA sulfate being $2,000 \%$, that of DA sulfate $180 \%$. DA sulfate has been shown to rise in plasma in parallel with plasma creatinine (6) and to be excreted in the isolated perfused kidney more slowly than free DA (7). DOPA sulfate may also be decarboxylated to DA sulfate (8). In HBP, a defective DOPA-DA conversion has been observed (3). The present data suggest defective decarboxylation also when HBP is complicated by CRF. The mechanism of this defect is unclear. HBP patients manifest a borderline decrease in plasma aromatic acid decarboxylase (AADC) activity (9). Such a defect can be accentuated in the presence of a decreased renal cell mass (10) which is the source of AADC regulating DOPA decarboxylation to DA.

We can conclude that in hypertensive patients with or without CRF, DOPA decarboxylation to DA is defective. Although basal free DOPA is not elevated, its progressive cumulative increase in the presence of CRF may be the source of augmented baseline DOPA sulfate and possibly also of heightened DA sulfate. DOPA sulfate accumulation is further accentuated in CRF following DOPA administration with predominance further skewed in favor of DOPA sulfate over DA sulfate (Fig. 2). While DOPA sulfate remains a biologically-inactive precursor pool, DA sulfate, as shown in some infusion studies of free DA (11), may be an endogenous inhibitor of free DA action and its excess may explain the decreased urinary free DA response to salt loading in CRF (12). Attenuated free DA-induced vasodilation in CRF (2) is suggested by its blunted BP-decreasing action in CRF (4). The strategically most important vascular DA effect may be its vasodilation of the vas efferens. Defective efferent vasodilation decreasing glomerular outflow due to free DA deficiency may contribute to glomerular hypertension (1) and thus perpetuate CRF complicating HBP.

\section{References}

1. Neuringer JR, Brenner BM: Glomerular hypertension: cause and consequences of renal injury. $J$ Hypertens 1992; 10 (suppl 7): S91-S97.

2. Smit AJ, Meijer S, Wesseling H, Reitsma WD, Donker AJM: Impaired renal hemodynamic but conserved natriuretic response to dopamine in patients with renal disease. Nephron 1989; 52: 338-346.

3. Kuchel O, Shigetomi S: Defective dopamine generation from dihydroxyphenylalanine in stable essential hypertensive patients. Hypertension 1992; 19: 634638.

4. Kuchel O, Shigetomi S: Dopaminergic abnormalities in hypertension associated with moderate renal insufficiency. Hypertension 1994; 23 (suppl I): I-240-I-245.

5. Cuche J-L, Prinseau J, Selz F, Ruget G, Baglin A: Plasma free, sulfo- and glucuro-conjugated catecholamines in uremic patients. Kidney Int 1986; 30: 566572.

6. Hashizume K, Ogihara T, Yamatodani A, Yamamoto $\mathrm{T}$, Wada H, Kumahara Y: Plasma levels and renal clearance of two isomers of dopamine sulfate in patients with essential hypertension. Clin Exp Hypertens 1988; 10: 561-574.

7. Buu NT, Duhaime J, Kuchel O: Handling of dopamine and dopamine sulfate by isolated perfused rat kidney. Am J Physiol 1986; 250: F975-F979.

8. Kuchel O, Buu NT, Racz K, De Léan A, Serri O, Kyncl J: Role of sulfate conjugation of catecholamines in blood pressure regulation. Fed Proc 1986; 45: 2254-2259.

9. Boomsma F, van der Hoorn FAJ, Schalekamp ADH: Determination of aromatic-L-amino acid decarboxylase in human plasma. Clin Chem Acta 1986; 159: 173-183.

10. Itskovitz HD, Wynn NC: Renal functional status and patterns of catecholamine excretion. J Clin Hypertens 1985; 3: 223-227.

11. Kuchel O, Buu NT, Serri O: Dopamine negatively modulates its own blood pressure increasing action. Can J Cardiol 1987; 3: 18-22.

12. Casson IF, Lee MR, Brownjohn AM, et al: Failure of renal dopamine response to salt loading in chronic renal disease. $\mathrm{Br}$ Med $J$ 1983; 286: 503-506.

13. Kuchel O: Clinical implications of genetic and acquired defects in catecholamine synthesis and metabolism. Clin Invest Med 1994; 17: 369-388. 